\title{
Enabling Robotic and Human Exploration: A Relay Network for the Future of Mars Exploration
}

\author{
A white paper submitted to the National Research Council \\ as input to the 2020 Planetary Decadal Survey
}

\author{
By Roy E. Gladden ${ }^{1}$, Charles H. Lee ${ }^{1}$, Charles D. Edwards ${ }^{1}$, \\ Michelle A. Viotti ${ }^{1}$, and Richard M. Davis ${ }^{2}$ \\ ${ }^{1}$ Jet Propulsion Laboratory, California Institute of Technology; \\ ${ }^{2}$ NASA Headquarters
}

9 July 2020

For additional information, please contact: Roy Gladden, roy.e.gladden@jpl.nasa.gov

The research was carried out at the Jet Propulsion Laboratory, California Institute of Technology, under a contract with the National Aeronautics and Space Administration (80NM0018D0004).

(c) 2020 All rights reserved.

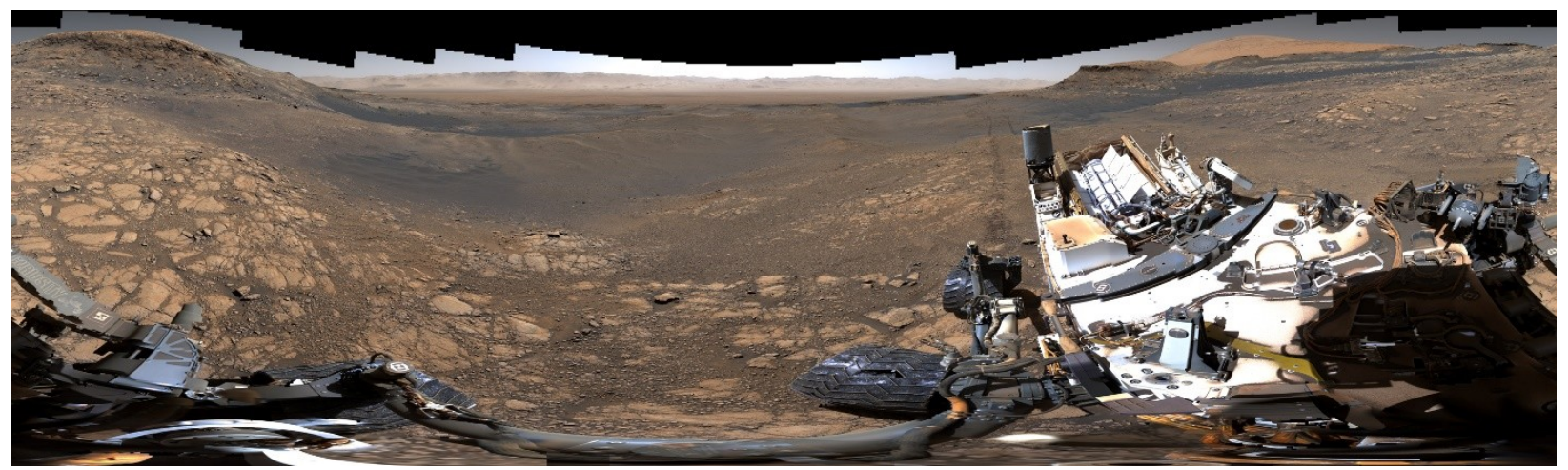

Figure 1: In 2019, NASA's Curiosity rover captured the highest resolution panorama yet of the Martian surface. The full image contains nearly 1.8 billion pixels. The Mars Relay Network returned every bit of the pre-processed images, after which ground operators assembled the panorama. The demand for high-resolution, data-intensive investigations will grow in the next decade to meet ambitious Agency science and reconnaissance goals. 


\subsection{Rationale: Why a Relay Network?}

A mission-enabling, next-generation relay capability at Mars is critical for supporting high-priority science and reconnaissance goals in the next decade and beyond. It would reduce the cost and risk of future Mars missions, relieving them of the need to carry their own direct-to-Earth communications systems (with attending mass and power requirements), and ensure an increased return on investment with greater data return from each mission. It would provide breakthrough increases in Mars telecommunication capabilities across a wide range of user spacecraft, with strategic benefits that include:

1. Continuous/near-continuous support to landers, rovers, and orbiters, with greatly increased instantaneous data rates and total data return. Currently, relay support to all surface assets returns a combined $\sim 1.4 \mathrm{~Gb} / \mathrm{sol}$ (via UHF), achieved via $\sim 6$ sporadic 10-30 minute relay sessions/sol; no relay services are available to low-altitude Mars orbiters. By contrast, a next-gen relay capability would provide continuous relay services at $200 \mathrm{~Gb} / \mathrm{sol}$ in the same time (via X-band) for most of the surface of Mars ( \pm 40 deg latitude). In addition, for the first time, high-rate relay services for low-altitude Mars orbiters could also be continuously provided. Aggregate data return for all Mars missions is currently $\sim 40 \mathrm{~Tb} / y e a r$, but this could be increased to over $1000 \mathrm{~Tb} / y e a r$ in service to both science and reconnaissance for human precursor missions.

2. Support to next-generation, data-intensive sensors. A next-gen relay capability would enable data-intensive investigations that have been unachievable to date. For example, mapping the spatial extent and depth of water ice in the near subsurface of Mars is a NASA goal for the next decade. This would require a synthetic aperture radar (SAR) and raw radar data returns that exceed present-day designs for directto-Earth communication systems. In addition, since late 2006, only a tiny fraction of Mars ( 4\%) has been mapped at HiRISE's $30 \mathrm{~cm} /$ pixel resolution at visible wavelengths. A next-gen relay network would enable both of these data-intensive objectives.

3. Support for high-resolution mapping of Mars at increased temporal resolution. Periodic imaging of the same surface features at high resolution would revolutionize the detection of changes on the surface of Mars (e.g., new impact craters, gullies/recurring slope lineae, dust devil tracks, ice caps, shifting dunes, etc.). A next-gen relay network would enable these science and reconnaissance investigations.

4. Support to orbital users, allowing low-cost "smallsat" orbiters to achieve "bigsat" science return. Currently, relay support for orbiter missions does not exist. As opportunities for low-cost and frequent access to Mars grows for NASA, other space agencies, and commercial partners, decadal-class science and reconnaissance capabilities become both affordable and achievable. Study results show that orbiters with an exceptionally modest telecom system would be able to achieve data returns more than twice that of the existing MRO downlink capability (see §2.1). This new class of relay service would enable emerging small orbital mission concepts freed of the need for high mass/power direct-to-Earth communication payloads and capable of decadal-class science at a fraction of the cost of today's large, complex orbiters. 
5. Support to surface users with increased ground-in-the-loop interactions at relatively short (10-40 $\mathrm{min}$ ) round-trip light times (RTLT). Currently, ground-in-theloop interactions, constrained by sporadic contact opportunities and the RTLT between Earth and Mars, account for a significant amount of the total time available for Mars surface operations. In contrast, a continuous relay capability would provide many Earth-Mars decision loops per sol, allowing for the better use of onboard resources and mission time (see \$2.2). Relay services could be provided for surface assets on a demand-access basis (i.e., only when conditions merit it), which allows simultaneous communications support for users without advanced planning.

Current Status: A highly successful international collaboration, the current "Mars Relay Network" (MRN) leverages the combined NASA and ESA orbiter capabilities to transfer data from Mars surface assets, and uses international protocol standards (see $\underline{1}, \underline{2}, \underline{3}$, and 4 ) to ensure interconnectivity. Since 2001, NASA's and ESA's efforts have collectively resulted in five orbiters and five landed missions to Mars meeting or exceeding their scientific objectives and planned mission lifetimes.

Due to the challenges of entry, descent, and landing (EDL), Mars landers and rovers are highly constrained in mass and volume, causing severe constraints on antenna size and transmission power, which in turn greatly reduces direct-to-earth (DTE) communications capabilities in comparison to orbiters. The Opportunity rover's (2004-2018) DTE downlink had only $1 / 600^{\text {th }}$ the downlink capability of MRO (operating since 2006). To address this problem, Mars surface spacecraft use higher data rates over short-range links to nearby Mars orbiters, which then transmit the data over the long distance to Earth. This relay strategy offers a variety of key benefits to landed assets, including increased data return, reduced energy requirements, reduced communications system mass, increased communications opportunities, robust critical event communications, and in situ navigation.

To date, the combined relay capabilities of the MRN orbiters have relayed and returned over 2.6 terabits of data from NASA assets on the surface of Mars, representing over $99 \%$ of acquired scientific data (Figure 2). Notably, the Phoenix lander did not have a direct-to-Earth communications capability, and relied wholly on the MRN for surface operations. Though these past missions have achieved great success through the MRN, nextdecade missions in planning (e.g., Ice Mapper) for both science and reconnaissance are expected to have greater communication needs; this would also provide redundancy for the planned Mars Sample Return

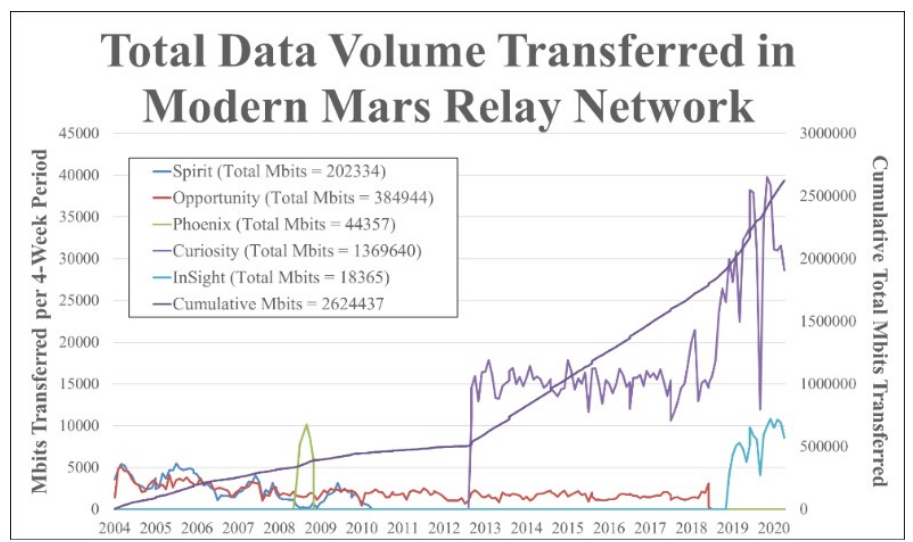

Figure : Data volume transferred via the Mars Relay Network, 2004-2020. The short Phoenix mission is evident (green spike), as is the dramatic increase in data volume returned after the Curiosity rover arrived at Mars in 2012. Data volume returned increased again when TGO came online in 2018. missions. Building a next-generation relay capability is an investment that provides es- 
sential capacity for the next decade and for increased human and robotic missions thereafter.

\subsection{Driving Near-Term Need \#1: An Aging Relay Infrastructure}

The Mars Exploration Program Advisory Group (MEPAG) expressed a major concern about the future of Mars exploration: the continued viability of the aging orbiters as relay assets. It "recommends NASA pursue innovative ways to refresh the orbital relay and reconnaissance capacity at Mars to support ongoing (e.g., Mars 2020) and new landed science and enabling both orbital and landed science. This may well be enabling for the future use of small spacecraft and competed missions (e.g., InSight) to study Mars." The last orbiter to join the MRN was ESA's ExoMars Trace Gas Orbiter (TGO) in 2016. The oldest active spacecraft in the network remains the 2001 Mars Odyssey, acting continuously as a relay service provider since 2004. Remaining network orbiters are operating beyond their primary design lifetimes, and few are expected to survive to 2030.

Principally designed for science missions, these orbiters addressed relay requirements as a secondary function. For Odyssey, this operationally manifests as a restriction in available onboard data storage for relay. For MRO, several instruments cause significant electromagnetic interference, which inhibits relay on the primary communications frequency. MAVEN's spacecraft design includes both a body-fixed UHF antenna for relay and a body-fixed DTE antenna, requiring the entire spacecraft to slew to communicate. Though the relay community has learned to work with these limitations, relay planning activities and the gathering of science data is nonetheless constricted.

\subsection{Driving Near-Term Need \#2: A Dedicated Relay Infrastructure}

In the short-term, the scientific community, universities, nation states (with both mature and nascent space programs), and private entities desire to send missions large and small to Mars for both science and reconnaissance. In the longer-term, a yearning exists to send humans to Mars to explore and, ultimately, to stay. The ability to communicate with Earth remains one of the principal technical challenges of all Mars missions (along with safely delivering missions to Mars and ensuring power for them). Existing assets on the surface of Mars have a very limited ability to communicate directly with Earth due to the extreme range and the limited mass and power that could be safely delivered to the surface. Partly for this reason, small science missions (diggers, climbers, cave explorers, weather stations, mini-rovers, balloons, drones, swarms, etc.) remain impractical. Even though the current MRN might provide sporadic support, relay opportunities are short and the amount of data throughput remains limited.

Currently, every Mars orbiter carries its own independent high-rate communications system. The attending large mass and power requirements constrain the ability of these orbiters to perform mapping, reconnaissance, monitoring, and other science data-collecting functions. As human precursor missions begin to manifest, this communications challenge will compound unless a generalized communications architecture is instantiated. A dedicated relay communications infrastructure would reduce the cost and risk of future robotic and human Mars missions and increase the return on investment, as measured by greater data return. This infrastructure should provide reliable, continuous/near-continuous support to surface and in-orbit users, greatly in- 
creased instantaneous data rates for proximity links at Mars, and vastly higher Mars-toEarth data rates, offering next-generation "trunk line" capabilities.

\subsection{Solution: A Small Constellation of Commsats}

As numerous studies of Mars communications architecture options have been conducted, this white paper focuses on a parametric analysis ("the Study") performed by the Mars Program Office at NASA's Jet Propulsion Laboratory (April 2018). The Study assessed the potential relay service characteristics of a small constellation of a new class of dedicated Mars telecommunications relay orbiters (commsats). The intent was to explore key design parameters for this relay constellation and to quantify performance benefits.

The Study assumed three equallyequipped commsats, ${ }^{1}$ spread uniformly in a circular equatorial orbit (Figure 3). While the Study examined a wide range of scenarios and commsat altitudes, results discussed here focus on commsats in a 6000 $\mathrm{km}$ altitude orbit. $^{2}$ Notably, larger and more powerful antennas anywhere in these scenarios would achieve a greater data throughput in the results.

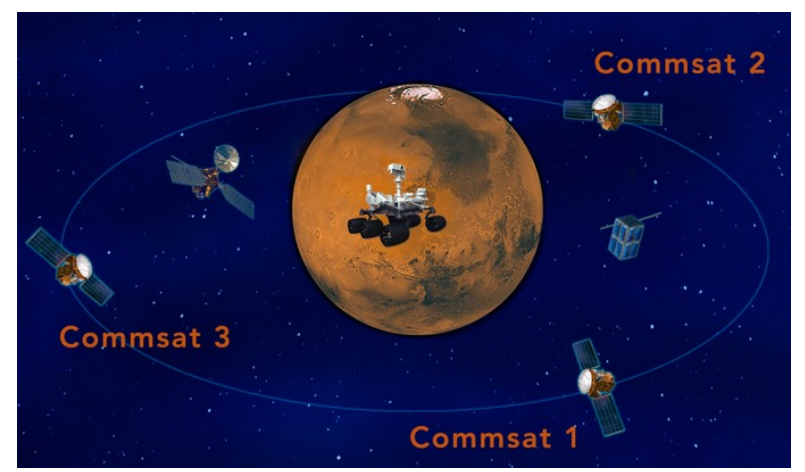

Figure 3: General architecture of proposed relay network.

\subsection{Enabling Orbiter Missions}

Modeled in $400 \mathrm{~km}$ orbits about Mars, the Study assumed two orbiter classes communicating via the commsats for 8 hours per day, and compared the results to the real-world experience of MRO, which carries the largest telecom system of any spacecraft at Mars.

\begin{tabular}{|c|c|c|}
\hline $\begin{array}{l}\text { Figure 4: Orbiter Mission } \\
\text { Scenarios }\end{array}$ & Scenario & Results \\
\hline \multirow{3}{*}{$\begin{array}{l}\text { Orbiting Users } \\
\text { (in } 8 \text { hours per day) }\end{array}$} & Baseline: $\quad$ MRO direct-to-Earth & 24 Gb/day \\
\hline & Orbiter A: $1 \mathrm{~W}, 10 \mathrm{~cm} \mathrm{X-band} \mathrm{antenna}$ & 50 Gb/day \\
\hline & Orbiter B: 15 W, $30 \mathrm{~cm} \mathrm{X-band} \mathrm{antenna}$ & 1200 Gb/day \\
\hline
\end{tabular}

Analogous to a very small spacecraft, Orbiter A carried a $1 \mathrm{~W}, 10 \mathrm{~cm}$, steerable X-band antenna. The Study showed that this very small orbiter could achieve data returns more than twice that of MRO using its exceptionally modest telecom system. Orbiter A's primary operational cost would be tracking the commsats with its small antenna, a technical capability not significantly different than tracking Earth.

Orbiter B carried a larger $15 \mathrm{~W}, 30 \mathrm{~cm}$, steerable X-band antenna, a system that was still quite modest relative to MRO's DTE payload (100 W, $3 \mathrm{~m})$. The Study showed that

\footnotetext{
${ }^{1}$ The Study was rather broad and necessarily made numerous assumptions about the analyzed scenarios, radios, margins, encoding schemes, etc. The Study did not address specific design considerations for the commsats, such as pointing capabilities, antenna designs, spacecraft power, onboard data handling, frequency allocation and utilization, etc.

${ }^{2}$ This representative altitude provides continuous communications coverage to a single landed asset near the Martian equator, guarantees that each commsat may view each other (to support cross-link communications), and ensures that a single 34-m Deep Space Network (DSN) antenna on Earth can simultaneously service all 3 commsats, with all visible to Earth almost $90 \%$ of the time. 
the data return capabilities represent a new class not previously achieved at Mars. This type of data throughput would enable high-resolution imaging for mapping, weather monitoring, and other purposes, not only for science data acquisition, but also in support of reconnaissance and eventual human efforts at Mars. Indeed, such a satellite could itself be used as a relay asset for surface users (see §2.3).

The Study demonstrated that the commsats would be mission-enabling for small science missions, returning "bigsat" data volumes in a "smallsat" form function.

\subsection{Enabling Landed Missions}

The Study included two main scenarios for landed missions near the equator and compared the results to the amount of data returned from the Curiosity rover through the MRN today during roughly 30 minutes of transmission time per sol.

\begin{tabular}{|l|l|c|}
\cline { 2 - 3 } $\begin{array}{c}\text { Figure 5: Lander Mission } \\
\text { Scenarios }\end{array}$ & \multicolumn{1}{c|}{ Scenario } & Results \\
\hline \multirow{2}{*}{$\begin{array}{c}\text { Landed Users } \\
\text { (in } 30 \text { min per sol) }\end{array}$} & Baseline: Curiosity Rover via MRN & $0.9 \mathrm{~Gb} / \mathrm{sol}$ \\
\cline { 2 - 3 } & Lander A: Fixed 10 W, omni, UHF antenna & $\mathbf{0 . 2 5} \mathbf{~ G b / s o l}$ \\
\cline { 2 - 3 } & Lander B: Steered 15 W, 30 cm, X-band antenna & $\mathbf{5 0 ~ G b / s o l ~}$ \\
\hline
\end{tabular}

Analogous to the present-day Curiosity rover, Lander A carried a $10 \mathrm{~W}$, omni-directional UHF antenna. Lander B carried a $15 \mathrm{~W}, 30 \mathrm{~cm}$, steerable X-band antenna, similar in size and power to that used by the Curiosity rover, but tasked for communicating with the commsats rather than directly to Earth. ${ }^{3}$

When compared to the current MRN, the Study showed that landed users with communications capabilities similar to existing surface assets, such as Lander A, would not actually benefit from the high-altitude commsats due to the long slant ranges. However, the continuous availability of the commsats would enable a variety of previously unfeasible mission types. Presently, surface spacecraft must wait for MRN orbiters to be overhead. With the commsats, a surface user could access the network at operationally preferred times, such as when it has sufficient data (or power), when a notable event occurs, or when it needs instruction from remote operators. New mission classes could be realized with increased opportunities for ground-in-the-loop interactions at relatively short (10-40 min) RTLTs and many Earth-Mars decision loops per day.

In comparison, landed users with a steerable X-band antenna, such as Lander B, would clearly benefit from the presence of the commsats. Not only would they take advantage of the same operational flexibility described above, they could also yield 50 times the data return. This throughput would require the landed asset to carry steerable antennas, which current landed assets already have for direct-to-Earth communications. Applying current technologies could achieve greater data returns and improve mission flexibility.

\subsection{Useful Augmentations}

Many future science mission concepts include very small and cost-effective Mars spacecraft that may be unable to carry a steerable antenna. These missions would

\footnotetext{
${ }^{3}$ The data rate from Curiosity using its X-band antenna ranges between 500 bps to $32 \mathrm{kbps}$. As shown here, the current MRN far outstrips its unaided capability to return data to Earth, demonstrating the value of the relay network. 


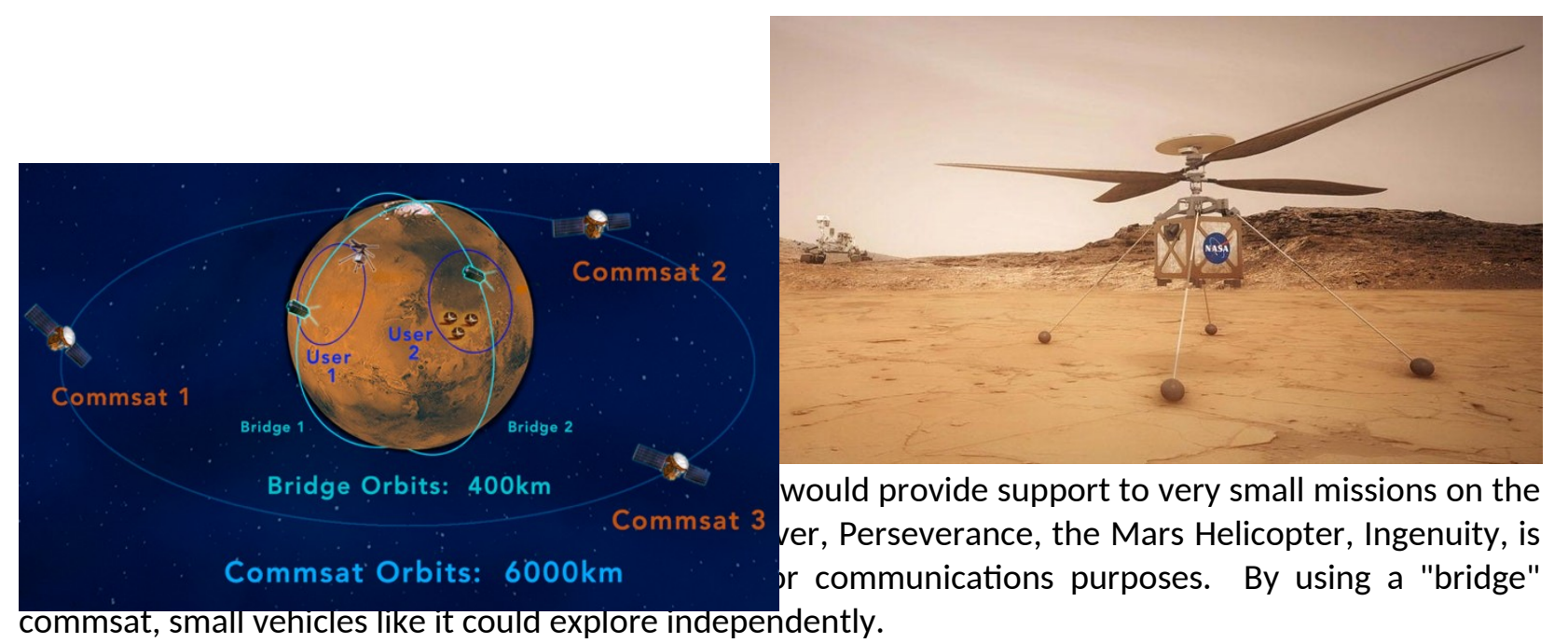

commsat, small vehicles IIke it could explore independently.

benefit from lower-altitude "bridge" commsats (perhaps placed in sun-synchronous, near-polar orbits) to connect low-power communications systems on the surface of Mars to the highly capable commsats.

The Study noted the challenge of providing relay services to more than one user at a time when each commsat carries a single steerable proximity-link antenna. Relay services would need to be pre-coordinated across the network, a challenge familiar to participants in the current MRN. To enable greater flexibility, commsats augmented with omnidirectional receivers could listen for a relay service request, allowing surface assets to make such requests only when conditions merit it (i.e. on a demand-access basis) and thus support multiple users without advanced planning.

Implementation of delay-tolerant networking protocols, defined by international standards, would also be enabling; data transfer could be managed by the network without human interaction (see also $\underline{5}$ ). Such methodology renders the network agnostic to the implementing organization and the vagaries of the user community, making it a truly generalized solution for use by any spacecraft that meets the interface specifications.

The commsats could provide other services, including time correlation and synchronization services, and radiometric services for position determination and navigation.

An atmospheric monitoring payload with a full-disk view of Mars could continuously observe the Martian meteorological system, continuing the now 21-year history of measuring and monitoring the atmosphere of Mars. This data would provide critical data to improve atmospheric models in support of the planned Mars Sample Return missions and future human exploration (i.e., for EDL, surface operations, and ascent operations).

A single commsat would return the data volumes identified herein every day regardless of the distance between Earth and Mars, suggesting that commsat cross-links may not be necessary for a small number of relay service users. However, a cross-link capability could split data into multiple data streams to reduce the transfer time to Earth, mitigate against Mars occultations, and provide service to multiple users.

As throughput demands are expected to increase dramatically due to reconnaissance and eventual human activities at Mars, more commsats than the three described would naturally be more enabling. In service to a larger community of relay users, such comm- 
sats could be deployed to a variety of orbits more optimal for data return at specific latitudes, for operational simplicity, or for increased network-level flexibility.

\subsection{Recommendations: A Mars Relay Infrastructure for Robotic and Human Activities}

The scientific and eventual human exploration of Mars will be hindered without a robust telecommunications infrastructure. With such an infrastructure in place, a greater variety of surface and orbiting spacecraft could be sent to Mars to realize new, exciting, and innovative missions. Instantiating even a modest set of commsats (e.g., the three described) would lay the foundation for an even larger and more robust network in the future, in service of an increased robotic and human presence at Mars. This pioneering effort would be a key component in advancing humanity's interests at the Red Planet.

\section{RECOMMENDATIONS FOR CONTINUING NASA'S LEGACY OF LEADING THE WORLD IN ADVANCING HUMAN INTERESTS IN MARS EXPLORATION}

Priority 1: At the earliest opportunity, establish a stable, next-generation network of dedicated commsats at Mars with payloads and orbits designed for their relay function (i.e., not as a minimal add-on to a primary science orbiter) in support of data-intensive investigations that have been unachievable to date, yet are critical to future missions.

- For the planned next-decade Mars missions (i.e. Mars Sample Return and Mars Ice Mapper) and all Mars assets, mission success is potentially risked without this capability, given the aging assets of the current MRN. The likely data-return needs of Mars Ice Mapper's synthetic aperture radar (SAR) could make this mission a near-term opportunity to establish a next-gen MRN for its own needs and that of other missions.

- Higher orbits offer breakthrough increases in availability for surface users, and, for the first time, relay support for orbiters. The infusion of state-of-the-art capabilities (e.g., Ka-band DTE links) would increase aggregate bandwidth and thus the amount and cost-effectiveness of science and reconnaissance returns.

- Cost-sharing and ridesharing opportunities should also be pursued via commercial and international partnerships to instantiate this network.

Priority 2: For the greatest return on investment, strategically consider how science- and reconnaissance-focused instruments and small missions could be deployed to achieve "bigsat" science via "smallsat" spacecraft, either as stand-alone missions that take advantage of the relay network or as part of the next-gen relay network itself.

- Such missions could include next-generation high-resolution, stereo imagers; weather instruments where a full-disk view of Mars and continuous data is needed; and, other data-intensive sensors for unprecedented spectral, spatial, and temporal resolution and high-data-rate returns for global coverage.

Priority 3: Promote the establishment of interoperability standards for com- 
munications and data systems across governmental and commercial organizations that would rely on next-gen relay capabilities.

- This network should take the lead in implementing international standards for space communications at Mars, particularly those related to Delay Tolerant Networking (DTN).

- Efforts by the Space Communication and Navigation (SCaN) Program and the Deep Space Network (DSN) to implement these standards in ground infrastructure should be supported. 